Revista

\title{
Multi-Ensayos
}

Vol. 6, núm. 11

ISSN: 2412-3285

https://multiensayos.unan.edu.ni

DOI: https://doi.org/10.5377/multiensayos.v6i11.9287

\section{Plan de mercadeo una oportunidad de aprendizaje de los mercados locales en la ciudad de Estelí}

\section{Marketing plan a learning opportunity for local markets in the city of Estelí}

Yasmina Ramírez Sobalvarro

Recibido: 24 de octubre de 2019. Aceptado: 13 de enero de 2020

\section{RESUMEN}

La asignatura de Plan de mercadeo es una nueva forma de visualizar la mercadotecnia dentro de la carrera, el grupo de IV año de mercadotecnia plan 16 es el primer grupo que logra desarrollar dicha materia. La mercadotecnia es una profesión dinámica y cada día se renueva como parte de la necesidad que tiene la misma para sobrevivir dentro la esfera de los negocios. En Nicaragua se ha convertido en una de las profesiones más populares en los últimos cinco años esto se evoca por la creciente ola de nuevos comercios que nacen cada día en nuestro país. El acceso a las redes de comunicación internacional, el uso del internet entre otros son algunos los factores que inciden directa e indirectamente. Pero realmente que es un Plan de mercadeo y porque es importante que los estudiantes de la carrera de Mercadotecnia lo logren hacer o desarrollar. Siendo el marketing un proceso responsable orientado a identificar, anticipar y satisfacer las necesidades del cliente, con la finalidad de fidelizarlo, de forma que la organización pueda obtener sus objetivos estratégicos.

Palabras claves: plan de mercadeo; estrategia; crecimiento de mercados; competencia.

\section{ABSTRACT}

Marketing plan subject is a new way to visualize marketing. The plan 16 group majoring in Marketing is the first group that completed this study subject in their IV year. Marketing is a dynamic profession, and every day it is renewed as part of the same need to survive within the sphere of business. In Nicaragua, it has become one of the most popular professions in the last five years. This is evoked by the growing wave of new businesses that emerge every day in our country. Access to international communication networks, the use of the Internet, among others, are some factors that directly and indirectly affect this area of study. Students majoring in Marketing must manage to write or develop a Marketing Plan, responsible process-oriented marketing to identify, anticipate, and meet the needs of clients and retain them so that a given business can achieve its strategic objectives.

Keywords: marketing plan; strategy; market growth; competition.

1 Docente titular en FAREM-Estelí. Correo electrónico: jazminara72@gmail.com, ORCID: https://orcid.org/0000-0002-4747-5658 (c) 2020 Revista Multi-Ensayos. 


\section{INTRODUCCIÓN}

En la actualidad, con mercados maduros y clientes informados y exigentes, se hace necesario que el responsable de marketing no sólo se ocupe de la dimensión operativa del mismo, sino también de su dimensión estratégica. Las estrategias de cartera, de segmentación y de posicionamiento se convierten en cuestiones clave; la competencia se intensifica y la innovación se acelera. Ya no es posible ofrecer productos que no guarden relación con lo que el mercado necesita, sino que resulta imprescindible actuar en función de lo que los clientes esperan de la empresa, con una perspectiva de fuera hacia dentro. Para realizar estas tareas, el responsable de marketing debe hacer del análisis la técnica de gestión más importante, con el fin de poder seleccionar opciones estratégicas sólidas en las que basar unos planes más eficientes de marketing operativo. Se trata no sólo de seguir la marcha del mercado, sino de anticiparse también a las necesidades cambiantes del cliente y adecuar la oferta a sus expectativas. Lo cual representa

Nos podemos preguntar dónde radica la importancia del Marketing, y su relación con el plan como tal Según lo expuesto en la literatura Marketing es según Peter Drucker.

"el marketing no es un departamento; es la empresa en su conjunto vista desde el punto de vista del cliente". Desde esta concepción, no es de extrañar que este prestigioso gurú de la gestión empresarial señalara que "dado que el papel de la empresa era crear clientes, sus dos únicas funciones esenciales eran el marketing y Ja innovación"( 1982)

Un Plan de mercadeo genera la oportunidad para que las empresas no solamente puedan conocer como están las ventas o los niveles de aceptación de sus productos y servicios en el mercado, sino que además genera la opción de poder realizar procesos de mejora continua, esto no solamente visto del enfoque de la competencia sino de la capacidad de respuesta que los clientes requieren la cual es cada vez más desafiante en el contexto de los mercados interactivos.

Para mejorar, las empresas deben de realizar periódicamente un análisis estratégico el cual servirá para determinar las cosas que se deben mejorar y aquellas que marchan de maravilla. Siempre pensando en incrementar la eficiencia de la organización y eficacia mediante el aumento de la capacidad de la organización para implementar sus recursos de forma inteligente.

\section{DESARROLLO}

La construcción de un plan de mercadeo representa la consecución de una serie de pasos entre los que se encuentran:

1. Análisis estratégico del entorno

2. Formulación de objetivos estratégicos

3. Construcción de una matriz de plan de acción

4. Creación de un presupuesto 
A continuación se describen cada uno de los acápites que lo conforman y la implementación de los mismos en MIPYMES locales.

\section{Análisis estratégico}

El análisis estratégico es el proceso que se lleva a cabo para investigar sobre el entorno de negocios dentro del cual opera una organización y el estudio de la propia organización, con el fin de formular una estrategia para la toma de decisiones y el cumplimiento de los objetivos.

El análisis estratégico sirve principalmente para que la empresa sepa hacia dónde quiere ir y hasta dónde quiere llegar. Las preguntas principales que una empresa debe plantearse al realizar un análisis estratégico son: ¿Cómo está constituido el mercado? ¿Cómo son los clientes activos en este sector?. Al hacer un análisis estratégico la empresa debe identificar el mercado sobre el que desea competir y así podrá definir sin problema una estrategia que la mantendrá presente en el rubro en el que participa.

El análisis estratégico debe de ser parte fundamental de las actividades de una empresa cada determinado tiempo, saber las cosas que está haciendo bien y aquellas que no están funcionando es muy importante para mantener su buen funcionamiento y organización (Certo \& Peters, 2015).

Se debe analizar a los clientes, sus motivaciones y necesidades insatisfechas. También se requiere identificar a la competencia, evaluar su desempeño, imagen, objetivos, estrategias, estructura, fortalezas y debilidades.

Se debe estar atentos a todos los factores externos que afectan una empresa, analizar el mercado, el crecimiento proyectado, tendencias, barreras, amenazas, áreas de oportunidades, y factores clave del éxito, etc.

\section{Pasos para realizar un análisis estratégico}

- Recopila datos específicos

- Analiza y evalúa tus procesos

- Desarrolla una serie de recomendaciones y presenta un plan de correcciones

- Monitorea los cambios y haz los ajustes

- Relaciónate con los involucrados para darle continuidad a las mejoras

\section{Los componentes del análisis estratégico son:}

1. Misión

2. Visión

3. Valores

4. Análisis FODA

5. Análisis del ciclo de vida del producto

6. Análisis de participación de mercado ( Matriz BCG)

7. Análisis de competencia ( Diamante de Porter) 


\section{Misión}

La declaración de Misión nos ayuda a mantener claridad proporcionando un marco de referencia para todas las decisiones que vayamos a tomar. Una Misión bien formulada no se debe confundir con los objetivos de la organización, la Misión trata sobre el beneficio que ofrecemos al cliente, se enfoca desde su perspectiva (Fred, 2011).

La declaración de Misión describe el concepto de la organización, la naturaleza de nuestros servicios, por qué estamos en él, a quién servimos y los valores bajo los cuales pretendemos funcionar. Una organización sin Misión no tiene identidad, ni un camino claro sobre el dirigir sus pasos. Utilizaremos una serie de preguntas genéricas que nos ayuden a identificar los aspectos a incluir en la declaración de Misión. Todas estas preguntas podrían resumirse sin dificultad en tres cuestiones básicas:

- ¿qué hacemos?,

- ¿para quién lo hacemos?,

- ¿cómo lo hacemos?

\section{La Visión}

Debe ser: breve, fácil de captar y recordar, inspiradora, planteando retos para su logro, creíble y consistente con los valores estratégicos y la misión, clara respecto a lo que debe llegar a ser la organización. La Visión tiene que transmitir y plantear retos para todos porque nos tiene que llevar a dónde queremos ir. Al igual que para la Misión, utilizaremos una serie de preguntas que nos ayuden a pensar mejor acerca de cómo debería ser el futuro de nuestra organización:

- ¿Qué es lo que yo veo como clave para el futuro de nuestra organización?,

- ¿Qué me emocionaría acerca de ser parte de esta organización en el futuro?

- ¿Qué valores necesitan ser acentuados?

\section{¿Qué valores necesitan ser acentuados?}

Definición de los objetivos Los objetivos son los resultados preestablecidos, más o menos alejados en el tiempo, que pretendemos alcanzar con nuestra propia acción. Determinan los resultados finales deseados y nos dicen exactamente dónde estamos y dónde queremos estar en un determinado momento. Forman una jerarquía que abarca desde aquéllos más amplios a los más específicos y, a su vez, éstos pueden subdividirse en objetivos más específicos, y así sucesivamente. Es lo que se conoce como pirámide de objetivos, donde los generales están articulados en torno a objetivos específicos. Esto permite que los objetivos más generales puedan alcanzarse a través de sus objetivos específicos, más operativos, y que los objetivos más específicos queden enmarcados por los objetivos más generales. El objetivo más complejo y ambicioso se vuelve fácil de realizar gracias a su descomposición en objetivos más simples y accesibles. 


\section{Análisis FODA}

La técnica FODA se orienta principalmente al análisis y resolución de problemas y se lleva a cabo para identificar y analizar las Fortalezas y Debilidades de la organización, así como las Oportunidades (aprovechadas y no aprovechadas) y Amenazas reveladas por la información obtenida del contexto externo.

El FODA como técnica de planeación, permitirá contar con información valiosa proveniente de personas involucradas con la administración del negocio y que con su know how pueden aportar ideas inestimables para el futuro organizacional. Es necesario señalar que la intuición y la creatividad de los involucrados es parte fundamental del proceso de análisis ya que para los que una determinada situación parece ser una oportunidad, para otros puede pasar desapercibida (Ponce, 2016)

\section{El ciclo de vida de los productos}

Existe una larga controversia acerca de la utilidad del ciclo de vida en el análisis estratégico, debido a la multiplicidad de formas posibles que puede adoptar esta curva, la longitud de las fases y los criterios de identificación de las fases, entre otros aspectos (Agueda,E; García, J; Narros, M.J; Olarte,C; Reinares, E;Saco,M, 2017).

Los productos se comportan como seres vivos y tienen un ciclo de vida que se divide en cuatro fases: nacimiento, crecimiento, madurez y declive. La evolución de las ventas del tamaño del mercado de un producto en función del tiempo presenta el comportamiento de acuerdo con las fases siguientes:

Fase I. Producto recién nacido (inicio). La empresa que dispone de un avance de tecnología o de unos grandes conocimientos de producción puede disfrutar de un cuasi monopolio. En general, encuentra problemas de puesta a punto y de lanzamiento al mercado. En esta fase, la empresa debe realizar inversiones importantes ya que el producto cuesta más de lo que rinde y es necesario soportar las pérdidas. En esta fase, la innovación es la ventaja competitiva determinante.

Fase II. Producto en pleno desarrollo (crecimiento). La empresa debe hacer esfuerzos de inversión todavía más importantes, ya sea para entrar en el mercado, ya sea para que la parte que ya ocupa no disminuya en beneficio de sus competidores, cada vez más numerosos. Esta fase se caracteriza por un gran aumento de los fondos en circulación y por unos beneficios escasos o inexistentes, ya que el rendimiento del crecimiento sirve para financiar la inversión.

En esta fase, la capacidad financiera para continuar la expansión es la ventaja comparativa determinante.

Fase III. Producto en la edad madura (madurez). El crecimiento del mercado disminuye y la empresa puede limitar sus inversiones a la renovación o a la mejora de los procesos. La competencia, muy viva, impone más que nunca sus exigencias para mantener o aumentar la parte del mercado. Las empresas competitivas obtienen beneficios, mientras que las otras están condenadas a tener pérdidas y, finalmente, 
a retirarse del mercado. En esta fase, la diferencia entre los competidores la establecen la calidad de los productos y de los servicios de distribución (Godás, 2011).

Fase IV. Producto que envejece (declive). Únicamente algunas empresas pueden subsistir en el mercado y un repliegue bien organizado no excluye beneficios importantes en la medida en que los productos vendidos están fabricados con equipos amortizados mucho tiempo antes. Para estas empresas, la clave de la rentabilidad pasa por la racionalización máxima de la producción y de la distribución.

\section{Análisis de la cartera de negocios (Matriz BCG)}

Las matrices de cartera o las matrices estratégicas representan un instrumento útil para el análisis estratégico en el que se combinan aspectos derivados del análisis externo y del análisis interno, de forma simultánea. Su denominación se debe a que estos modelos realizan el análisis para cada producto o grupo de productos de la empresa, contemplando a ésta como una cartera de negocios (Czinkota, M., Dickson, P., Dunne, P., Griffin , A., Hoffman, D. K., Hutt, M., 2011).

\section{Modelo del Boston Consulting Group (BCG) ${ }^{1}$}

Este modelo, al igual que los demás, que analizaremos, ha revolucionado la planificación estratégica durante los últimos veinte años. Todos ellos han proporcionado una fuente de información para facilitar la eliminación de los negocios débiles y el esfuerzo de utilizar convenientemente los recursos. El BCG proporciona un sistema útil para el análisis de un todo dentro de la suma de las partes componentes para determinar la extensión de la sinergia existente en él. Podemos analizar el modelo en base a tres factores de acción:

- "Performance" de grupo en términos del relativo "performance" de las empresas filiales.

- "Performance" del mercado en términos del relativo posicionamiento de los productos.

- "Performance" de ventas en términos del relativo aprovechamiento de los clientes.

El objetivo de este modelo es el de determinar la asignación más adecuada de recursos entre los distintos negocios de la empresa, identificando aquellos que son generadores de recursos y aquellos otros que absorben recursos.

Las dos dimensiones elegidas para el análisis son la tasa de crecimiento del mercado y la cuota relativa de mercado. La primera representa el atractivo actual y futuro de la industria, así como la necesidad de fondos por parte de la empresa para financiar inversiones que permitan mantener el ritmo de crecimiento de un negocio. Así, una tasa de crecimiento alta supone absorción de recursos para inversión. En la matriz, se distinguen dos zonas en función de esta dimensión según que se considere alta o baja la tasa de crecimiento. La línea divisoria entre ambas zonas no es fija y depende de la industria en la que se opere (Contreras, 2014).

1 Matriz de participación y cobertura de mercado 
La cuota relativa de mercado da una idea de la posición competitiva de la empresa indicando, asimismo, la capacidad de un negocio para generar recursos. Se define como la relación por cociente entre las ventas de la empresa y las de la mayor empresa competidora. Esta variable se representa en una escala logarítmica con la unidad como valor de referencia que separa la matriz en dos zonas que reflejan una posición fuerte o débil del negocio.

\section{Análisis del entorno competitivo (Diamante de Porter)}

El entorno competitivo de una empresa está constituido por un conjunto de actores y factores que ejercen una influencia directa sobre los resultados de la empresa y en los de sus competidores. El entorno competitivo es, pues, un elemento esencial para la empresa, por lo que su conocimiento y el estudio de su evolución son cuestiones clave a la hora de pasar a diseñar una estrategia de empresa Ahora bien, antes de iniciar su estudio es preciso realizar una serie de puntualizaciones relativas a los términos entorno competitivo, estructura de mercado y sector (Porter, 1995).

Al estudio de los distintos componentes del entorno competitivo se ha dedicado la economía industrial, si bien el estudio de cada componente se solía realizar de forma autónoma y sobre todo sin conexión con su influencia en la estrategia de empresa a elegir. Es con Porter (1982) cuando los componentes del entorno competitivo se estudian conjuntamente y en relación con sus implicaciones sobre la estrategia de empresa. De acuerdo con Porter estos elementos componentes del entorno competitivo o fuerzas competitivas se pueden agrupar en cinco conjuntos, los cuales aparecen representados en la siguiente figura

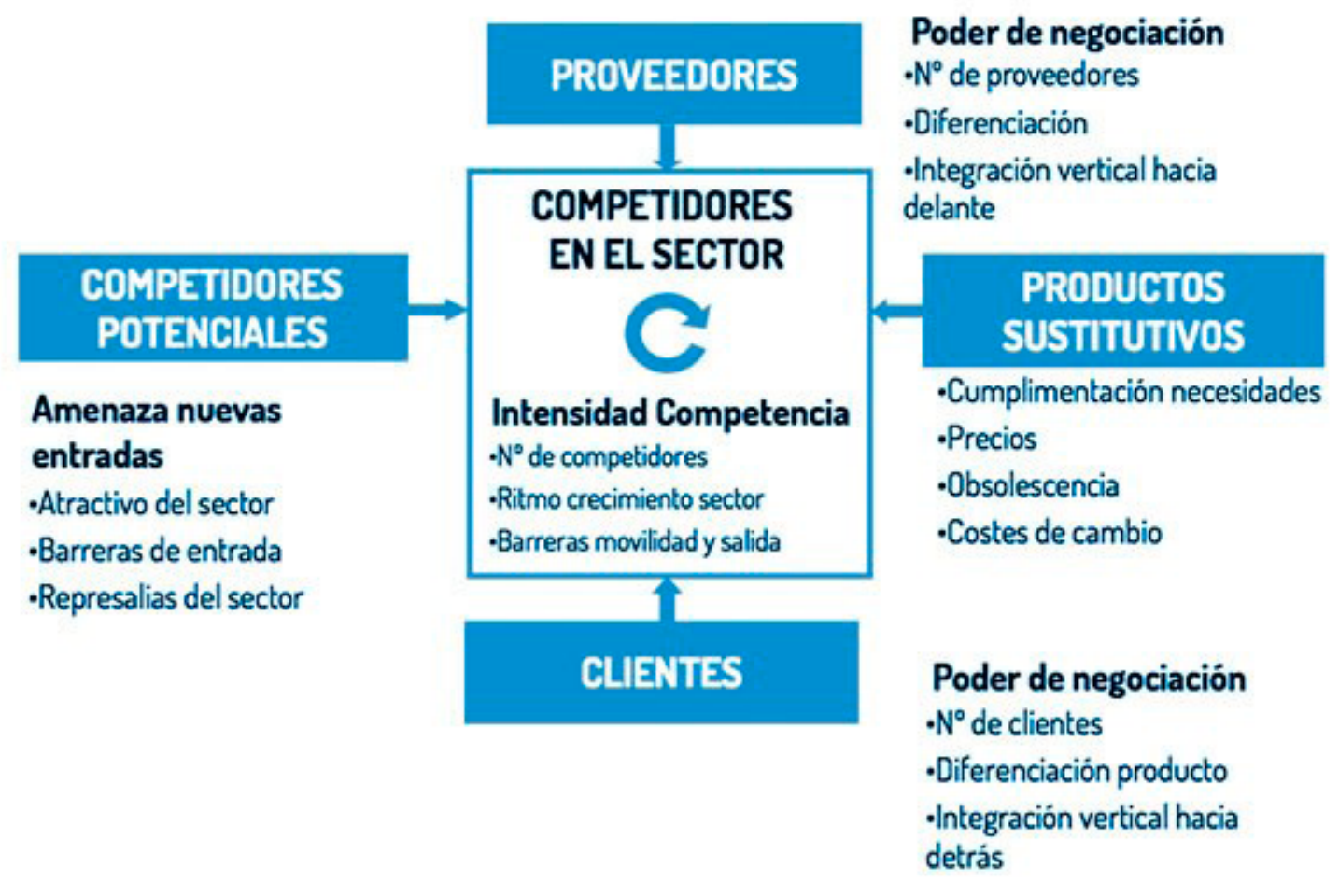

Fig. 2.1. Elementos componentes del entorno competitivo. Fuente: Adaptación de Porter. (1982): Estrategia Competitiva, pág.24 


\section{Definición de los objetivos de la empresa}

A nivel global, normalmente se estudian tres niveles de objetivos empresariales: La misión u objetivo supremo. Es la expresión muy general de lo que quiere ser la empresa, de sus aspiraciones en cuanto a su papel en la sociedad. Viene determinada por la filosofía, el sistema de valores y creencias imperantes en la empresa, los cuales son productos de la historia, heredados de sus fundadores y de los artífices de los grandes cambios de rumbo de la empresa si los hay, y la ética ante los clientes, competidores, empleados y la sociedad en general, influyendo de forma decisiva sobre el tipo de organización que la empresa quiere ser y el tipo de relaciones que quiere mantener con su entorno La misión de la empresa se entiende aquí como el resultado de la cultura de la empresa.

\section{Los objetivos deben ser SMART}

- Específicos (Specific): Claros sobre qué, dónde, cuándo y cómo va a cambiar la situación;

- Medibles (Measurable): que sea posible cuantificar los fines y beneficios;

- Realizables (Achievable): que sea posible lograr los objetivos (conociendo los recursos y las capacidades a disposición de la comunidad);

- Realistas (Realistic): que sea posible obtener el nivel de cambio reflejado en el objetivo;

- Limitado en tiempo (Time bound): estableciendo el periodo de tiempo en el que se debe completar cada uno de ellos.

\section{El Plan de acción}

El plan de acción es un trabajo en equipo, por ello es importante reunir a los demás trabajadores comunitarios y a los miembros de la comunidad y formalizar el grupo llamándolo "Comité de planeamiento" u otra denominación.

El plan lleva los siguientes elementos

- Que se quiere alcanzar (objetivo)

- Cuánto se quiere lograr (cantidad y calidad)

- Cuándo se quiere lograr (en cuánto tiempo)

- En dónde se quiere realizar el programa (lugar)

- Con quién y con qué se desea lograrlo (personal, recursos financieros)

- Cómo saber si se está alcanzando el objetivo (evaluando el proceso)

- Cómo determinar si se logró el objetivo (evaluación de resultados)

Los planes de acción solo se concretan cuando se formulan los objetivos y se ha seleccionado la estrategia a seguir. Los principales problemas y fallas de los planes se presentan en la definición de los detalles concretos. 
Para la elaboración del plan es importante identificare las grandes tareas y de aquí desglosar las pequeñas. Se recomienda utilizar un "cuadro de plan de acción" que contemple todos los elementos.

Los componentes de la estrategia y los objetivos estratégicos se ventilaron en la unidad anterior, ahora corresponde visualizar los componentes restantes.

$\begin{array}{lllll}\text { Estrategia } & \text { Objetivo estratégico } & \text { Tácticas o acciones } & \text { Presupuesto } & \text { KPI }\end{array}$

El plan de acción es un instrumento para la evaluación continua de un programa. Es a su vez la representación real de las tareas que se deben realizar, asignando responsables, tiempo y recursos para lograr un objetivo.

Toda empresa, grande, mediana o pequeña, que produzca y/o comercialice productos o servicios, adopta necesariamente, una serie de decisiones tácticas para guiar y controlar las acciones que realizará, con el fin de mantener, con la mayor probabilidad de éxito, sus relaciones con el o los mercados a los que se dirige. Las metas se derivan de los objetivos y las tácticas se derivan de las estrategias. Una estrategia puede implantarse por medio de diferentes tácticas, pero una táctica sólo puede corresponder a una estrategia. El proceso global del marketing estratégico está compuesto por varias áreas de actividad:

- Conocer el mercado.

- Conocer las capacidades de la empresa.

- Definir los objetivos y las estrategias.

- Implantar mediante tácticas, la estrategia.

- Verificar los resultados: Retroalimentación

\section{El presupuesto publicitario en el plan de mercadeo}

El presupuesto publicitario es, como su propio nombre indica, la cantidad de dinero destinada a las inversiones en publicidad. A veces estos importes pueden parecer difíciles de establecer.

Hay que tener en cuenta que la parte que más recursos se van a llevar es la inserción de los anuncios en los diferentes medios de comunicación. ¿Qué hace que una campaña publicitaria tenga éxito?

Pero gastar en publicidad no es sólo insertar anuncios en TV, prensa o radio y ya está. Los anuncios también hay que idearlos y crearlos, realizar una investigación de mercado previa, etc. Por tanto, a los costes antes señalados hay que añadir los costes que habrá que pagar a la agencia de publicidad que se encargue de la elaboración de la campaña. 
Destinar una cantidad de dinero que haya disponible en las cuentas de la empresa: Simplemente se destina un dinero a publicidad sin tener en cuenta ningún estudio previo. Esto puede provocar que la inversión sea o insuficiente o un derroche.

A continuación se comparte con en el contexto de la asignatura se lograron trabajar las propuestas de doce planes de mercadeo para igual número de empresas locales.

Entre las empresas señaladas se encuentran:

1. Calzado Arauz la casa de las fajas

2. Financiera Tu Crédito

3. Farmacia FARMAPLUS

4. Impresiones SHALOM

5. Sitio Web MI vida Mi estilo

6. Decoraciones Milys Place

7. Librería Kathangel

8. Bordados Nicaragua

9. Blessing Salón

10. Carbón Vegetal El Negrito

11. Trasporte San Antonio

12. Comidería El Centro de la Gastronomía

\section{CONCLUSIÓN}

La construcción de planes de Mercadeo se debe de considerar una oportunidad tanto en el ámbito teórico como en el práctico puesto que de esta forma los estudiantes realizan análisis centrados en el contexto. Para la Mercadotecnia la mejor forma de poder comprender el comportamiento del cliente y de los mercados mismos nace del trabajo de campo y la articulación con las experiencias de los empresarios locales, esto es el sustento de un buen plan de mercadeo.

Para toda MIPYME ${ }^{2}$ indistintamente del ciclo de vida en el que se encuentre, el poder diseñar un plan de mercadeo genera no solo un estudio de la realidad circundante sino que además facilita los procesos competitivos al corto y mediano plazo.

La experiencia de facilitar procesos de Plan de mercadeo aunados al mercadeo estratégico genero la sensación de la misión cumplida, puesto que los estudiantes han logrado articular los conocimientos teóricos de una forma sencilla pero utilizando herramientas de cálculo en Excel y poder hacer análisis que da una mirada de conjunto sobre el desarrollo empresarial nivel local.

2 Pequeña y mediana empresa 


\section{REFERENCIAS}

Agueda,E; García, J; Narros, M.J; Olarte,C; Reinares,E;Saco,M. (2017). Principios de marketing. Madrid España.: editorial ESIC. Recuperado el 30 de junio de 2019

Certo, \& Peters, J. (2015). Dirección estratégica (3 ed.). Bogotá, Colombia: Mc Graw Hil. Recuperado el 02 de Septiembre de 2019

Contreras, J. (03 de marzo de 2014). 12manager. Recuperado el septiembre 01 de 2019, de 12manager: www.12manager.com

Czinkota, M., Dickson, P., Dunne, P., Griffin , A., Hoffman, D. K., Hutt, M. (2011). Principios de marketing y sus mejores prácticas. México, D.F: Cengage Learning Latin American. Recuperado el 30 de junio de 2019

Fred, R. D. (2011). Conceptos de Administración estratégica. México: Prentice Hall. Recuperado el 02 de Septiembre de 2019

Godás, L. (2011). El cilo de vida del producto. Elsevier, 110-115. Recuperado el 02 de septiembre de 2019

Ponce, H. (2016). La matriz FODA:una alternativa para realizar diagnósticos y determinar estrategias de intervención en las organizaciones productivas y sociales. Contribuciones a la Economía. Recuperado el 25 de junio de 2019

Porter, M. E. (1995). Estrategias competitivas. México: Continental. Recuperado el 25 de junio de 2019 